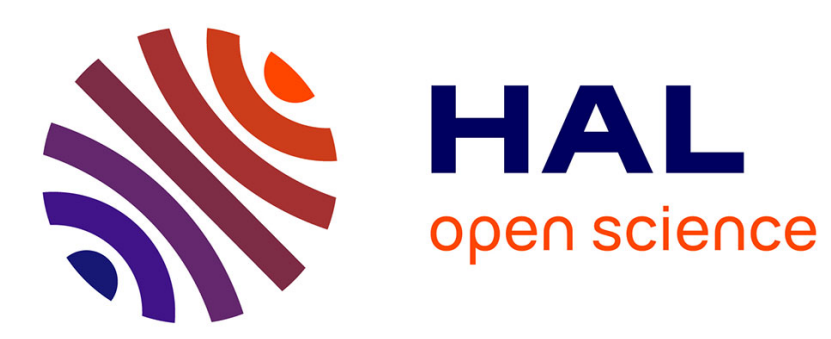

\title{
Embedding of Uncertainty Propagation: Application to Hierarchical Performance Analysis
}

\author{
M Dinh, Anton Korniienko, Gérard Scorletti
}

\section{To cite this version:}

M Dinh, Anton Korniienko, Gérard Scorletti. Embedding of Uncertainty Propagation: Application to Hierarchical Performance Analysis. 5th Symposium on System Structure and Control, IFAC, IFAC, Feb 2013, Grenoble, France. pp.190-195, 10.3182/20130204-3-FR-2033.00165 . hal-01181166

\author{
HAL Id: hal-01181166 \\ https://hal.science/hal-01181166
}

Submitted on 29 Jul 2015

HAL is a multi-disciplinary open access archive for the deposit and dissemination of scientific research documents, whether they are published or not. The documents may come from teaching and research institutions in France or abroad, or from public or private research centers.
L'archive ouverte pluridisciplinaire HAL, est destinée au dépôt et à la diffusion de documents scientifiques de niveau recherche, publiés ou non, émanant des établissements d'enseignement et de recherche français ou étrangers, des laboratoires publics ou privés. 


\title{
Embedding of Uncertainty Propagation: Application to Hierarchical Performance Analysis
}

\author{
M. Dinh ${ }^{*}$ A. Korniienko ${ }^{* *}$ G. Scorletti ${ }^{* *}$ \\ *31000 Toulouse, France (e-mail: marc.dinh@gmail.com) \\ ** Ecole Centrale de Lyon, 69134 Ecully Cedex, France (e-mail: \\ anton.korniienko@ec-lyon.fr, gerard.scorletti@ec-lyon.fr)
}

\begin{abstract}
The article proposes an approach to embed the propagation of structured uncertainty through a system in a system with one unstructured uncertainty. This embedding is interesting as it allows to use the classical analysis and design tools such $H_{\infty}$ design and $\mu$-analysis or synthesis. A strong motivation is to improve the computational efficiency of the analysis for large-scale uncertain systems using a hierarchical approach. Examples are provided for both the embedding in the SISO and MIMO cases and for the hierarchical performance analysis of an uncertain Phase-Locked-Loop network.
\end{abstract}

Keywords: robustness analysis, hierarchical system, finite dimensional LMI.

\section{INTRODUCTION}

In the 80's-90's, $\mu$-analysis (Doyle (1982); Skogestad and Postlethwaite (2005)) was developed to investigate the $\left(H_{\infty}\right)$ performance of Linear Time Invariant systems in the case of structured uncertainties. This approach is based on the computation of the structured singular value $\mu$ of frequency dependent complex matrices, which was proved to be NP-hard by Braatz et al. (1994). Fortunately, lower and upper bounds on $\mu$ can be efficiently computed, the computation of the $\mu$ upper bound (Fan et al. (1991)) allowing to guarantee a certain level of performance, with some conservatism. A major interest of the use of $\mu$ upper bound is to obtain a satisfying trade-off between the complexity of the computation, i.e. the efficiency, and the conservatism of the obtained result.

Nevertheless, even if the computation is efficient, the computation time can be important in the case of largescale uncertain systems. The purpose of the paper is to propose a robust analysis method with a trade-off between efficiency and conservatism adapted to large-scale systems. Our motivating application is the robustness analysis of Phase-Locked-Loop (PLL) networks (Korniienko et al. (2011a)), a challenging problem in Microelectronics.

In the case of large-scale systems with uncertainties defined by cones, Safonov (1983) proposed to perform a hierarchical analysis using a propagation of conic uncertainty result. Unfortunately, its direct application to a (largescale) system with structured uncertainties could lead to an overly conservative result. In this paper, we propose a new hierarchical analysis method which is adapted to the case of uncertain systems with structured uncertainties.

In our approach, the large-scale uncertain system is represented as an interconnection of sub-systems with structured uncertainties. We propose $(i)$ to compute for each uncertain sub-system a "good" unstructured uncertainty, in the sequel, referred to as the embedding, (ii) to apply $\mu$-analysis to the interconnected system where each sub-system is replaced by the unstructured uncertainty. With respect to the direct application of the $\mu$-analysis to the large-scale uncertain system, the computation is performed in two sequential steps. In the first step, the computation of the embeddings of the sub-systems can be parallelized. In our motivating application (PLL network), the sub-systems are actually the same. Furthermore, the computation of $\mu$ in the second step is more efficient than the computation of $\mu$ for the original system.

In order to apply this approach, we first investigate the computation in step $(i)$. Note that this problem is a generalization of the propagation of conic uncertainties presented in Safonov (1983). More precisely, for a given frequency, how to compute the "best" unstructured uncertainty that embeds an uncertain system with structured uncertainties? Beyond the hierarchical analysis application, the solution to this problem has many interesting applications. For instance, in the Quantitative Feedback Theory (Houpis and Rasmussen (1999)), the aim is to design a controller that achieves some level of performances in front of all the uncertainties in the system. In the method, it is assumed that it is possible to know the propagation of the uncertainty through the system. Another interesting application is the use of the embedding to perform $\mu$-synthesis in the integrated framework by Yaw and Balas (2011); the advantage being the computation time thanks to the embedding.

Our solution is based on a separation of graph theorem. First proposed in Safonov (1980) as a general approach to feedback system analysis, specialized forms were proposed in e.g. (Iwasaki and Hara (1998); Scorletti (1998)) for (uncertain) LTI system analysis. Since the $\mu$ upper bound proposed in Fan et al. (1991) can be interpreted as a particular application of the separation of graph theorem, this 
theorem was applied to extend $\mu$-analysis to time-delay systems in Scorletti (1998) or to time-varying/nonlinear systems in Megretski and Rantzer (1997), to reduce the conservatism of the $\mu$ upper bound Scorletti et al. (2007), to cite a few. In this paper, we reveal another interesting application of this powerful theorem.

Some notations and preliminary on the separation of graph are first provided in Section 2. Section 3 presents the embedding result and the hierarchical analysis approach. Numerical examples are detailed in Section 4.

\section{NOTATIONS AND PRELIMINARY}

\subsection{Notations}

$\mathbb{R}^{n}$ (resp. $\mathbb{C}^{n}$ ) denotes real (resp. complex) vectors of size $n . \mathbb{R}^{m \times n}$ (resp. $\mathbb{C}^{m \times n}$ ) denotes real (resp. complex) matrices of size $m \times n$. $I_{n}$ denotes the identity matrix of size $n .0_{m \times n}$ denotes the null matrix of size $m \times$ $n$. When the subscript are omitted, $I$ and 0 denotes the identity and null matrices of appropriate size. $X^{*}$ denotes the transpose conjugate of $X$ while $X^{+}$denotes its Moore-Penrose inverse. $X<0$ stands for $\mathrm{X}$ is definite negative. stands for the Rhedeffer star product and $\otimes$ for the Kronecker product. The notation $\mathbf{b d i a g}_{i}\left(T_{i}\right)$ or bdiag $\left(T_{1}, \cdots, T_{N}\right)$ represents the block diagonal matrix of the form:

$$
\left[\begin{array}{ccc}
T_{1} & 0 & 0 \\
0 & \ddots & 0 \\
0 & 0 & T_{N}
\end{array}\right]
$$

\subsection{Mathematical preliminary on separation of graph}

The results obtained in the paper are derived from the following theorem, see for instance Iwasaki and Hara (1998).

Theorem 1. Let $\Delta \subset \mathbb{C}^{n_{o} \times n_{i}}$ be a compact set and $M$ be a complex matrix partionned into $\left[\begin{array}{ll}A & B \\ C & D\end{array}\right]$ with $A \in \mathbb{C}^{n_{i} \times n_{o}}$ and $D \in \mathbb{C}^{n_{z} \times n_{w}}$. Let $P_{p} \in \mathbb{C}^{n_{z} \times n_{z}}$.

Then the inequality

$$
\forall \Delta \in \Delta,(\Delta \star M)^{*} P_{p}(\Delta \star M)<0
$$

holds if and only if there exists a matrix $\Phi \in \mathbb{C}^{\left(n_{o}+n_{i}\right) \times\left(n_{o}+n_{i}\right)}$ such that

$$
\begin{gathered}
\forall \Delta \in \Delta,\left[\begin{array}{c}
\Delta \\
I
\end{array}\right]^{*} \Phi\left[\begin{array}{c}
\Delta \\
I
\end{array}\right] \geq 0 \\
{\left[\begin{array}{l}
C^{*} \\
D^{*}
\end{array}\right] P_{p}\left[\begin{array}{ll}
C & D
\end{array}\right]+\left[\begin{array}{cc}
I & 0 \\
A & B
\end{array}\right]^{*} \Phi\left[\begin{array}{cc}
I & 0 \\
A & B
\end{array}\right]<0}
\end{gathered}
$$

Note that if $\Delta \star M$ represents the graph of a system $G$, that is $\Delta \star M=\left[\begin{array}{l}N \\ D\end{array}\right]$ with $G=N D^{-1}$, then (1) defines a dissipativity property on $G$.

\section{EMBEDDING AND HIERARCHICAL ANALYSIS}

\subsection{Problem formulation}

As we use $\mu$-analysis in the second step of the proposed hierarchical approach, a frequency gridding is defined so that we are concerned with uncertain matrices. The problem of interest is then the embedding of an uncertain matrix with structured uncertainty into an uncertain matrix with a direct additive unstructured uncertainty.

Problem 1. (Embedding Problem). Let the set of matrices $G_{s} \subseteq \mathbb{C}^{n_{z} \times n_{w}}$ be defined by a set of structured uncertainty $\boldsymbol{\Delta}_{\mathbf{s}}$, a compact subset of $\mathbb{C}^{n_{o} \times n_{i}}$, and a complex matrix $M_{s}$ partitionned into $\left[\begin{array}{ll}A_{s} & B_{s} \\ C_{s} & D_{s}\end{array}\right]$ with $A_{s} \in \mathbb{C}^{n_{i} \times n_{o}}$ and $D_{s} \in \mathbb{C}^{n_{z} \times n_{w}}$ such that

$$
G_{s}=\left\{\Delta_{s} \star M_{s}, \Delta_{s} \in \Delta_{\mathbf{s}}\right\}
$$

Find $G_{\text {nom }} \in \mathbb{C}^{n_{z} \times n_{w}}, W_{i} \in \mathbb{C}^{n_{w} \times n_{w}}$ and $W_{o} \in \mathbb{C}^{n_{z} \times n_{z}}$ that defines $G_{u}$, the set of matrices

$$
G_{u}=\left\{G_{n o m}+W_{o} \Delta_{u} W_{i}, \bar{\sigma}\left(\Delta_{u}\right)<1\right\}
$$

such that $G_{s} \subseteq G_{u}$.

Assumptions: we assume that $W_{i}$ and $W_{o}$ are invertible.

\subsection{Main result}

Two equivalent results can be obtained.

Theorem 2. Let $\boldsymbol{\Delta}_{\mathbf{s}} \subset \mathbb{C}^{n_{o} \times n_{i}}$ be a compact set and $M_{s}$ be a complex matrix partitionned as stated in the Embedding Problem.

There exist:

- a matrix $G_{n o m} \in \mathbb{C}^{n_{z} \times n_{w}}$;

- invertible matrices $W_{o} \in \mathbb{C}^{n_{z} \times n_{z}}$ and $W_{i} \in \mathbb{C}^{n_{w} \times n_{w}}$;

such that $G_{s} \subseteq G_{u}$ with $G_{s}$ and $G_{u}$ defined in equations (2) and (3) respectively if and only if there exists a solution to one of the two following problems:

(1) First: there exist

- a matrix $P_{n o m} \in \mathbb{C}^{n_{z} \times n_{w}}$;

- a positive definite matrix $P_{o} \in \mathbb{C}^{n_{z} \times n_{z}}$;

- a positive definite matrix $P_{i} \in \mathbb{C}^{n_{w} \times n_{w}}$;

- a matrix $\Phi \in \mathbb{C}^{\left(n_{o}+n_{i}\right) \times\left(n_{o}+n_{i}\right)}$; such that (4) and (5) are satisfied

$$
\begin{aligned}
& \forall \Delta_{s} \in \Delta_{\mathbf{s}}, \quad\left[\begin{array}{c}
\Delta_{s} \\
I
\end{array}\right]^{*} \Phi\left[\begin{array}{c}
\Delta_{s} \\
I
\end{array}\right] \geq 0 \\
& {[.]^{*}\left[\begin{array}{cc|c|c}
-P_{o} & I & 0 & P_{\text {nom }} \\
I & 0 & 0 & 0 \\
\hline 0 & 0 & \Phi & 0 \\
\hline-P_{\text {nom }}^{*} & 0 & 0 & -P_{i}
\end{array}\right]\left[\begin{array}{ccc}
I & 0 & 0 \\
0 & C_{s} & D_{s} \\
\hline 0 & I & 0 \\
0 & A_{s} & B_{s} \\
\hline 0 & 0 & I
\end{array}\right]<0}
\end{aligned}
$$

with $P_{o}=W_{o} W_{o}^{*}, P_{i}=W_{i}^{*} W_{i}$ and $P_{n o m}=G_{n o m}$.

(2) SECond: there exist

- a matrix $\widetilde{P}_{n o m} \in \mathbb{C}^{n_{z} \times n_{w}}$;

- a positive definite matrix $\widetilde{P}_{o} \in \mathbb{C}^{n_{z} \times n_{z}}$;

- a positive definite matrix $P_{i} \in \mathbb{C}^{n_{w} \times n_{w}}$;

- a matrix $\Phi \in \mathbb{C}^{\left(n_{o}+n_{i}\right) \times\left(n_{o}+n_{i}\right)}$;

such that (4) and (6) are satisfied

$$
[.]^{*}\left[\begin{array}{cc|c|c}
-\widetilde{P}_{o} & \widetilde{P}_{o} & 0 & \widetilde{P}_{\text {nom }} \\
\widetilde{P}_{o} & 0 & 0 & 0 \\
\hline 0 & 0 & \Phi & 0 \\
\hline-\widetilde{P}_{\text {nom }}^{*} & 0 & 0 & -P_{i}
\end{array}\right]\left[\begin{array}{ccc}
I & 0 & 0 \\
0 & C_{s} & D_{s} \\
\hline 0 & I & 0 \\
0 & A_{s} & B_{s} \\
\hline 0 & 0 & I
\end{array}\right]<0
$$

with $\widetilde{P}_{o}=W_{o}^{-*} W_{o}^{-1}, P_{i}=W_{i}^{*} W_{i}$ and $\widetilde{P}_{n o m}=$ $\widetilde{P}_{o} G_{n o m}$. 
Table 1. Possibilities of minimization

\begin{tabular}{|c||c|c|c|}
\hline Name & Minimization & Constraint & Solution \\
\hline \hline $\mathrm{c} 1$ & $\bar{\sigma}\left(P_{i}\right)$ & $\bar{\sigma}\left(P_{o}\right)<c$ & 1 \\
\hline $\mathrm{c} 2$ & $\bar{\sigma}\left(P_{i}\right)$ & $\left\|P_{o}\right\|_{F}<c$ & 1 \\
\hline $\mathrm{c} 3$ & $\left\|P_{i}\right\|_{F}$ & $\bar{\sigma}\left(P_{o}\right)<c$ & 1 \\
\hline $\mathrm{c} 4$ & $\left\|P_{i}\right\|_{F}$ & $\left\|P_{o}\right\|_{F}<c$ & 1 \\
\hline \hline $\mathrm{c} 5$ & $\bar{\sigma}\left(P_{o}\right)$ & $\bar{\sigma}\left(P_{i}\right)<c$ & 1 \\
\hline $\mathrm{c} 6$ & $\bar{\sigma}\left(P_{o}\right)$ & $\left\|P_{i}\right\|_{F}<c$ & 1 \\
\hline $\mathrm{c} 7$ & $\left\|P_{o}\right\|_{F}$ & $\bar{\sigma}\left(P_{i}\right)<c$ & 1 \\
\hline c8 & $\left\|P_{o}\right\|_{F}$ & $\left\|P_{i}\right\|_{F}<c$ & 1 \\
\hline \hline $\mathrm{c} 9$ & $-\log \left(\operatorname{det}\left(\tilde{P}_{o}\right)\right)$ & $\bar{\sigma}\left(P_{i}\right)<c$ & 2 \\
\hline $\mathrm{c} 10$ & $-\log \left(\operatorname{det}\left(\tilde{P}_{o}\right)\right)$ & $\left\|P_{i}\right\|_{F}<c$ & 2 \\
\hline
\end{tabular}

Proof. See the appendix for the complete proof.

\subsection{Discussion}

Theorem 2 presents a semi-infinite LMI optimization problem: constraint (4) defines an infinite number of constraints that cannot be solved efficiently as it. This constraint can be verified a priori by choosing the structure of the matrix $\Phi$ adequately. In this case, Theorem 2 defines a finite dimensional LMI optimization problem that can be solved efficiently. Moreover the equivalence can be preserved, depending on the nature of the $\boldsymbol{\Delta}_{\mathbf{s}}$ set.

For instance, a standard set $\boldsymbol{\Delta}_{\mathbf{s}}$ is

$\left\{\operatorname{bdiag}_{j}\left(\delta_{j} I_{n_{j}}\right)\right.$, with $\delta=\left[\delta_{i}\right] \in \mathbb{R}^{r}$ such that $\left.\|\delta\|_{\infty} \leq 1\right\}$ representing parametric uncertainties. $\Phi$ can then be chosen of the form

$$
\left[\begin{array}{cc}
-D & G \\
G^{*} & D
\end{array}\right]
$$

where $D=\operatorname{bdiag}_{k}\left(D_{k}\right)$, with $D_{k}=D_{k}^{*}>0$, and $G=\operatorname{bdiag}_{k}\left(G_{k}\right)$, with $G_{k}=-G_{k}^{*}$. This corresponds to the usual $D$ - $G$ scalings of the $\mu$-analysis (Fan et al. (1991)).

Scorletti et al. (2007) introduced the $L$ scaling to reduce the conservatism in the case of $r \geq 2$. Indeed, it is possible to represent the set $G_{s}$ with a non standard set $\boldsymbol{\Delta}_{\mathrm{s}}$ (and the appropriate matrix $M_{s}$ ) of the form

$$
\left\{\delta \otimes I, \text { with } \delta=\left[\delta_{i}\right] \in \mathbb{R}^{r} \text { such that }\|\delta\|_{\infty} \leq 1\right\} .
$$

$\Phi$ can then be chosen of the form

$$
\Phi=\left[\begin{array}{cc}
-D+j L & G \\
G^{*} & \sum D_{i}
\end{array}\right] \text { with } L=\left[\begin{array}{cccc}
0 & V_{1,2} & \ldots & V_{1, r} \\
-V_{1,2} & 0 & \ddots & \vdots \\
\vdots & \ddots & \ddots & V_{r-1, r} \\
-V_{1, r} & \ldots & -V_{r-1, r} & 0
\end{array}\right]
$$

where $D=\operatorname{bdiag}_{k}\left(D_{k}\right)$, with $D_{k}=D_{k}^{*}>0, G=$ $\left[\ldots G_{k} \ldots\right]$, with $G_{k}=-G_{k}^{*}$, and $V_{i, j}=V_{i, j}$ are real matrices.

It is interesting to obtain an embedding of minimum size. Matrix norms, such as the maximum singular value or the Frobenius norm, can be used as an indicator of size. Another interesting indicator is the $\log (\operatorname{det}()$. one which corresponds to the volume of the underlying ellipsoid. Adding this minimization still leads to finite dimensional LMI problems, when using the corresponding solution in Theorem 2. Table 1 presents the possible combinations. From this table, we do not expect any difference in the results between combinations $\mathrm{c} 1$ and $\mathrm{c} 5$, neither between combinations $\mathrm{c} 4$ and $\mathrm{c} 8$. We also expect some symmetry between c2 and c7 and between c3 and c6. Moreover, the maximum singular value being a worst case in all directions, we expect the Frobenius norm and the $\log (\operatorname{det}()$.$) indicators to be better. For the SISO case,$ all combinations are equivalent.

Direct multiplicative uncertainty at input can be deduced from the direct additive uncertainty studied here. The embedding set is

$$
\left\{G_{\text {nom }}^{i}\left(I+W_{o}^{i} \Delta_{u} W_{i}^{i}\right), \bar{\sigma}\left(\Delta_{u}\right)<1\right\}
$$

which is the same set as the additive one with $G_{n o m}=$ $G_{n o m}^{i}, W_{o}=G_{n o m}^{i} W_{o}^{i}$ and $W_{i}=W_{i}^{i}$. In the case when $G_{n o m}$ is full column rank, $W_{o}^{i}$ can be computed by $G_{n o m}^{+} W_{o}$. Similarly, it is possible to deduce a direct multiplicative uncertainty at output from the direct additive one.

\subsection{Hierarchical analysis}

An interesting application of the previous result is the problem of the performance analysis of linear large-scale uncertain systems. A large-scale uncertain system is a system composed of uncertain sub-systems which are interconnected according to a given topology. It can be represented by a feedback of the sub-systems $T_{i}$ regrouped into a block diagonal operator $T$ and an interconnection operator $M$, see Fig. 1 and equation (9).

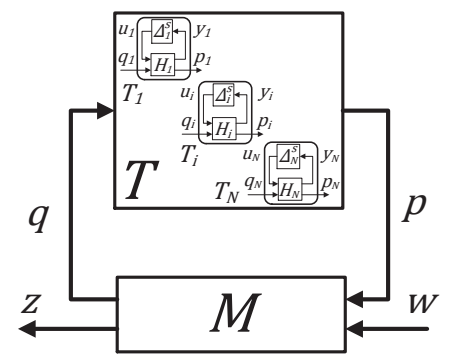

Fig. 1. Global uncertain linear large-scale system

$$
\left[\begin{array}{c}
q \\
z
\end{array}\right]=M\left[\begin{array}{c}
p \\
w
\end{array}\right] \text { and } p=\overbrace{\operatorname{bdiag}_{i}\left(T_{i}\right)}^{T} q
$$

with the dimensions of the vectors $q, p, z$ and $w$ equal to $n_{q}, n_{p}, n_{z}$ and $n_{w}$.

Each sub-system $T_{i}$ is an interconnection of the nominal sub-system part $H_{i}$ and the uncertainty $\Delta_{i}^{s}$ belonging to the structured set $\boldsymbol{\Delta}^{\mathbf{s}}{ }_{i} \subseteq \mathbb{C}^{n_{\text {out }}^{i} \times n_{\text {in }}^{i}}$ which definition is similar to the set $\boldsymbol{\Delta}_{\mathbf{s}}$ :

$$
\left[\begin{array}{c}
y_{i} \\
p_{i}
\end{array}\right]=H_{i}\left[\begin{array}{l}
u_{i} \\
q_{i}
\end{array}\right] \text { and } u_{i}=\Delta_{i}^{s} y_{i}
$$

with the dimensions of the vectors $q_{i}, p_{i}, u_{i}$ and $y_{i}$ equal to $n_{q}^{i}, n_{p}^{i}, n_{u}^{i}$ and $n_{y}^{i}$.

The following formalization of the performance analysis problem is proposed.

Problem 2. For a given large-scale system defined by (9) and (10), a given frequency gridding vector $v_{\omega}=\left\{\omega_{0}\right\}$ and given structured uncertainty sets $\boldsymbol{\Delta}^{\mathbf{s}}{ }_{i}$ for all $i \in\{1, \ldots, N\}$ find a frequency depending upper bound $\left\{\gamma\left(\omega_{0}\right)\right\}$ on the $\left(H_{\infty}\right)$ performance transfer function between global external input $w$ and output $z$ signals. 
As discussed in the introduction, the proposed approach is composed of two steps:

(1) the first step based on the given result (Theorem 2) allows to define the unstructured embedding similar to (3) of the structured uncertainty $\Delta_{i}^{s}$ of each subsystem;

(2) replacing the sub-system dynamics $T_{i}$ by their embedding and transforming the global system into a standard $M-\Delta$ form results in a new global system representation given in Fig. 2 and described by:

$$
\left[\begin{array}{c}
\widetilde{q} \\
z
\end{array}\right]=\widetilde{M}\left[\begin{array}{c}
\tilde{p} \\
w
\end{array}\right] \text { and } \widetilde{p}=\overbrace{\operatorname{bdiag}_{i}\left(\Delta_{i}^{u}\right)}^{\Delta^{u}} \widetilde{q}
$$

with the dimensions of the vectors $\widetilde{q}, \widetilde{p}, z$ and $w$ equal to $n_{q}, n_{p}, n_{z}$ and $n_{w}$ and the uncertainty $\Delta_{i}^{u}$ belonging to the unstructured set $\boldsymbol{\Delta}^{\mathbf{u}}{ }_{i}=\mathbb{C}^{n_{\text {out }}^{i} \times n_{\text {in }}^{i}}$ and such that $\bar{\sigma}\left(\Delta_{i}^{u}\right)<1$. Further application of the standard $\mu$-analysis tools accomplishes the second step and thus resolve Problem 2, with a different trade-off between complexity and conservatism compared to a direct structured $\mu$-analysis.

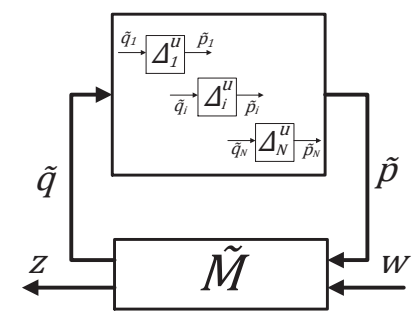

Fig. 2. Unstructured embedding of global uncertain linear large-scale system

The trade-off of the proposed approach can be changed in the first step by using several kinds of embedding (sector, ellipsoid, circle, half-plan, etc...). Note that the computation of the embeddings can be parallelized so that this first step does not need more time while decreasing the conservatism. It is also pointed out that the proposed approach is particularly interesting in the case of homogenous large-scale systems with sub-systems having a common nominal part $H_{1}=H_{2}=\cdots=H_{N}$ and all the structured uncertainties belonging to the same set. The computed embeddings are then valid for all the subsystems, decreasing the computational load.

The trade-off is also dependent on the way how a largescale system is decomposed into the structure of Fig. 1, which is not unique. For some systems, as for the PLL network, the decomposition is natural and fully exploits the homogeneous nature of the sub-systems. Moreover, there can be more than one hierarchical level.

\section{NUMERICAL EXAMPLES}

\subsection{Local uncertainty propagation: SISO embedding case}

We begin with a scalar case. The main advantage is that it is simple to understand and, more importantly, to evaluate. Indeed, the embedding will be a disk of center $G_{n o m}$ and of radius $\left|W_{o} W_{i}\right|$.

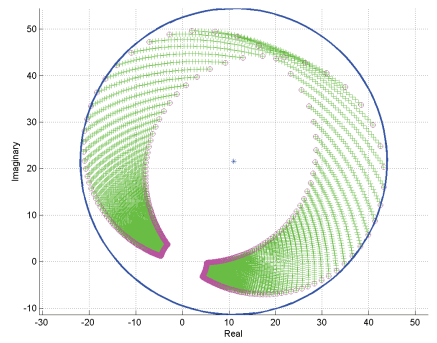

Fig. 3. Visualisation for SISO embedding example

SISO EXAMPLE: the scalar with structured uncertainty under study is

$$
G^{S I S O}\left(j \omega_{0}\right)=\frac{-\omega_{0}^{2}+2 \xi_{n} \omega_{n}\left(j \omega_{0}\right)+\omega_{n}^{2}}{-\omega_{0}^{2}+2 \xi_{d} \omega_{d}\left(j \omega_{0}\right)+\omega_{d}^{2}}
$$

with $\omega_{0}=1 \mathrm{rad} / \mathrm{s}, \xi_{n}=0.7$ and $\xi_{d}=0.01$. The uncertainties are real and are defined by $\omega_{n} \in(0.1,0.5)$ and $\omega_{d} \in(0.9,1.1)$. In the sequel, a gridding will be performed on $\left[\omega_{n} \omega_{d}\right]^{T}$, with a step of 0.01 on $\omega_{n}$ and with a step of 0.001 on $\omega_{d}$.

The structured uncertain set $\boldsymbol{\Delta}_{\mathrm{s}}$ is then

$$
\left\{\left[\begin{array}{cc}
\omega_{n} I_{2} & 0 \\
0 & \omega_{d} I_{2}
\end{array}\right] \mid \omega_{n} \in(0.1,0.5), \omega_{d} \in(0.9,1.1)\right\} .
$$

By scaling $\omega_{n}$ and $\omega_{d}$, the matrix $\Phi$ can be of the form defined in (7). The results show that it is not necessary to use the $D-G-L$ scalings of (8).

SISO EMBEDDING EvAluATion: as foreseen, for all the combinations, we obtain

$$
\begin{aligned}
G_{\text {nom }}^{\text {SISO }} & \approx 11.015+j \times 21.51 \\
\left|W_{o}^{S I S O} W_{i}^{\text {SISO }}\right| & \approx 32.95
\end{aligned}
$$

Fig. 3 represents the Nyquist response and allows a visualization of the embedding: the embedding disk encompasses all the values that can take $G^{S I S O}$ with the gridding defined. For this example, the embedding is excellent.

\subsection{Local uncertainty propagation: MIMO embedding case}

MIMO EXAMPLE: for the MIMO case, we choose the following example

$$
G^{M I M O}\left(j \omega_{0}\right)=\operatorname{odir} \times G^{S I S O}\left(j \omega_{0}\right) \times i d i r
$$

with odir $=\left[\begin{array}{ll}1 & -1.5\end{array}\right]^{T}$ and $i d i r=\left[\begin{array}{ll}1 & 2\end{array}\right]$. This example has the advantages that the matrix has a strong direction at input and output that can be evaluated.

MIMO EMBEDDING EvALUATION: for all the combinations, we get

$$
G_{n o m}^{M I M O} \approx \operatorname{odir} \times G_{n o m}^{S I S O} \times i \operatorname{dir}
$$

which is excellent.

The directionality can be evaluated by computing the singular value decomposition of $W_{o}^{M I M O}$ and $W_{i}^{M I M O}$. In this example, the directionality is taken into account if the ratio between the maximum and minimum singular values is large. These ratios are presented in Table 2. Note that $\log (\operatorname{det}()$.$) indicator takes into account the directionality$ extremely well. It is also the case for the Frobenius norm 
Table 2. Ratio of the singular values on the weightings

\begin{tabular}{|c||c|c|}
\hline & $W_{o}^{M I M O}$ & $W_{i}^{M I M O}$ \\
\hline \hline $\mathrm{c} 1$ & 1.2363 & 1.2245 \\
\hline $\mathrm{c} 2$ & 70.1072 & 1.2252 \\
\hline $\mathrm{c} 3$ & 1.2270 & 44.6502 \\
\hline $\mathrm{c} 4$ & 29.8985 & 29.2731 \\
\hline \hline $\mathrm{c} 5$ & 1.2300 & 1.2438 \\
\hline $\mathrm{c} 6$ & 1.2248 & 60.8302 \\
\hline $\mathrm{c} 7$ & 53.3795 & 1.2287 \\
\hline $\mathrm{c} 8$ & 29.8907 & 29.9473 \\
\hline \hline $\mathrm{c} 9$ & 3825 & 1.2237 \\
\hline $\mathrm{c} 10$ & 4744 & 9.9795 \\
\hline
\end{tabular}

indicator. However, the maximal singular value leads too very poor results from this perspective.

For the evaluation of the size, let us compute for each pair of combination $(i, j)$

$$
\max _{\bar{\sigma}\left(\Delta_{u}^{i}\right)<1} \bar{\sigma}\left(\left(W_{o}^{j}\right)^{-1} W_{o}^{i} \Delta_{u}^{i} W_{i}^{i}\left(W_{i}^{j}\right)^{-1}\right)
$$

where the superscript $M I M O$ have been dropped. This gives the minimum size of the unstructured uncertainty of the embedding $j$ for which the embedding $i$ is included in the embedding $j$. Table 3 presents these minimum sizes. As expected, c1 and c5 leads to very poor results as they are not included in any other combination whereas the other combinations are all included in $\mathrm{c} 1$ and $\mathrm{c} 5$. Note also that c10 leads to very good results as it is included in c1, c2, c5, c7 and c9, c4, c8 and c9 performs also quite well. However, what is surprising is that $\mathrm{c} 3$ and $\mathrm{c} 6$ are not included in c4 or $\mathrm{c} 8$.

\subsection{Global analysis: hierarchical analysis of a PLL network}

Let us consider now a numerical example of hierarchical performance analysis of a large-scale system. One takes as an example the performance analysis of the active clock distribution network from Korniienko et al. (2011a) subject to technological dispersions. An active clock distribution network is composed of $N=16$ mutually synchronized Phase-Locked-Loops (the sub-systems) delivering the clock signals to the chip. To be able to synchronize the PLLs exchange the information on their relative phase through the interconnection network and the phase detectors.

Since the principal aim of the system is the synchronization, the PLLs are identical or at least have a common nominal part. One has thus $H_{1}=H_{2}=\cdots=H_{N}$. Of course, during the manufacturing process, there are inevitable technological dispersions which can be represented in the form of parametric uncertainties belonging to the same set. We have thus $\forall i \in\{1, \ldots, N\}$ :

$$
T_{i}\left(j \omega_{0}\right)=\frac{k_{i}\left(j \omega_{0}+a_{i}\right)}{-\omega_{0}^{2}+k_{i} j \omega_{0}+k_{i} a_{i}}
$$

where $k_{i}, a_{i}$ are the real uncertain parameters defined as $k_{i} \in\left(0.76 \cdot 10^{4}, 6.84 \cdot 10^{4}\right)$ and $a_{i} \in(91.1,273.3) . \omega_{0}$ is the current frequency defined by gridding.

The exchange of information between the PLLs is modelled by an interconnection matrix $M$ defined in (13).

$$
M=\left[\begin{array}{ccccccccccccccccc}
0 & \frac{1}{3} & 0 & 0 & \frac{1}{3} & 0 & 0 & 0 & 0 & 0 & 0 & 0 & 0 & 0 & 0 & 0 & \frac{1}{3} \\
\frac{1}{3} & 0 & \frac{1}{3} & 0 & 0 & \frac{1}{3} & 0 & 0 & 0 & 0 & 0 & 0 & 0 & 0 & 0 & 0 & 0 \\
0 & \frac{1}{3} & 0 & \frac{1}{3} & 0 & 0 & \frac{1}{3} & 0 & 0 & 0 & 0 & 0 & 0 & 0 & 0 & 0 & 0 \\
0 & 0 & \frac{1}{2} & 0 & 0 & 0 & 0 & \frac{1}{2} & 0 & 0 & 0 & 0 & 0 & 0 & 0 & 0 & 0 \\
\frac{1}{3} & 0 & 0 & 0 & 0 & \frac{1}{3} & 0 & 0 & \frac{1}{3} & 0 & 0 & 0 & 0 & 0 & 0 & 0 & 0 \\
0 & \frac{1}{4} & 0 & 0 & \frac{1}{4} & 0 & \frac{1}{4} & 0 & 0 & \frac{1}{4} & 0 & 0 & 0 & 0 & 0 & 0 & 0 \\
0 & 0 & \frac{1}{4} & 0 & 0 & \frac{1}{4} & 0 & \frac{1}{4} & 0 & 0 & \frac{1}{4} & 0 & 0 & 0 & 0 & 0 & 0 \\
0 & 0 & 0 & \frac{1}{3} & 0 & 0 & \frac{1}{3} & 0 & 0 & 0 & 0 & \frac{1}{3} & 0 & 0 & 0 & 0 & 0 \\
0 & 0 & 0 & 0 & \frac{1}{3} & 0 & 0 & 0 & 0 & \frac{1}{3} & 0 & 0 & \frac{1}{3} & 0 & 0 & 0 & 0 \\
0 & 0 & 0 & 0 & 0 & \frac{1}{4} & 0 & 0 & \frac{1}{4} & 0 & \frac{1}{4} & 0 & 0 & \frac{1}{4} & 0 & 0 & 0 \\
0 & 0 & 0 & 0 & 0 & 0 & \frac{1}{4} & 0 & 0 & \frac{1}{4} & 0 & \frac{1}{4} & 0 & 0 & \frac{1}{4} & 0 & 0 \\
0 & 0 & 0 & 0 & 0 & 0 & 0 & \frac{1}{3} & 0 & 0 & \frac{1}{3} & 0 & 0 & 0 & 0 & \frac{1}{3} & 0 \\
0 & 0 & 0 & 0 & 0 & 0 & 0 & 0 & \frac{1}{2} & 0 & 0 & 0 & 0 & \frac{1}{2} & 0 & 0 & 0 \\
0 & 0 & 0 & 0 & 0 & 0 & 0 & 0 & 0 & \frac{1}{3} & 0 & 0 & \frac{1}{3} & 0 & \frac{1}{3} & 0 & 0 \\
0 & 0 & 0 & 0 & 0 & 0 & 0 & 0 & 0 & 0 & \frac{1}{3} & 0 & 0 & \frac{1}{3} & 0 & \frac{1}{3} & 0 \\
0 & 0 & 0 & 0 & 0 & 0 & 0 & 0 & 0 & 0 & 0 & \frac{1}{2} & 0 & 0 & \frac{1}{2} & 0 & 0 \\
0 & 0 & 0 & 0 & 0 & 0 & 0 & 0 & 0 & 0 & 0 & 0 & 0 & 0 & 0 & -1 & 1
\end{array}\right]
$$

In this example, the transfer function between external signals $w$ and $z$ expresses the performance of the global PLL network and namely its ability to synchronize with periodic reference signal $w$. This reference signal is represented by its phase so that the PLL network has to track a ramp. More details can be found in Korniienko et al. (2011b).

The proposed hierarchical analysis approach is applied. To decrease the conservatism, we use a matrix $\Phi$ of the form described in (8).

HiERARCHICAL ANALYSIS EVALUATION: the results are presented in the Fig. 4 where the nominal transfer function magnitude, the direct $\mu$-analysis upper bound and the hierarchical analysis upper bound are presented. Though the direct $\mu$-analysis upper bound is less conservative compared to the upper bound in the hierarchical case (maximum peak $13.4 d B$ and $6 d B$ respectively), the computation time is much more important in the first case $711 \mathrm{sec}$ compared to $55 \mathrm{sec}$. One can thus conclude that the hierarchical performance analysis can be efficiently applied in the case of large-scale system especially with a large number $N$ of sub-systems.

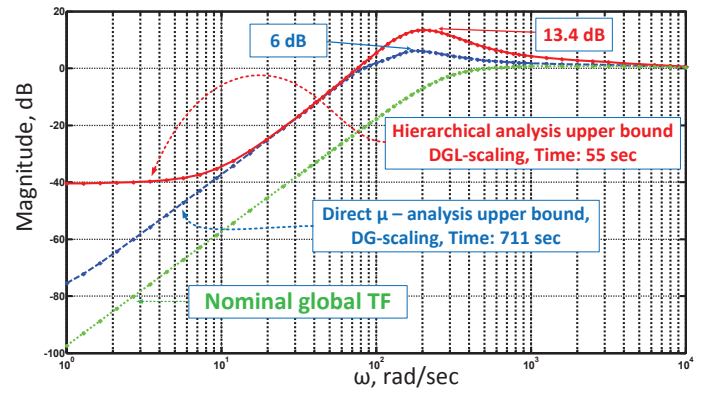

Fig. 4. Upper bounds for direct $\mu$-analysis case (blue dashed line) and Hierarchical analysis case (red full line), nominal performance transfer function (green dotted dashed line)

\section{CONCLUSION AND PERSPECTIVES}

An approach for embedding the propagation of structured uncertainty into an unstructured one was presented in 
Table 3. Minimum sizes of unstructured uncertainty for embedding

\begin{tabular}{|c|c|c|c|c|c|c|c|c|c|c|}
\hline${ }_{i}{ }^{j}$ & c1 & c2 & c3 & c4 & c5 & c6 & c7 & c8 & c9 & c10 \\
\hline c1 & 1 & 85.80 & 36.47 & 579.8 & 1.02 & 49.77 & 30.49 & 630.6 & 3228 & 44329 \\
\hline c2 & 1.00 & 1 & 36.47 & 23.94 & 1.02 & 49.74 & 1.01 & 25.25 & 37.67 & 517.2 \\
\hline c3 & 1.01 & 86.30 & 1 & 24.39 & 1.00 & 1.36 & 30.54 & 24.15 & 3241 & 10.212 \\
\hline $\mathrm{c} 4$ & 1.00 & 3.54 & 1.52 & 1 & 1.00 & 2.08 & 1.25 & 1.09 & 132.9 & 418.7 \\
\hline c5 & 1.03 & $\overline{86.09}$ & 36.90 & 573.6 & 1 & $\overline{49.02}$ & 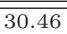 & 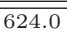 & 3233 & 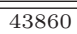 \\
\hline c6 & 1.01 & 86.46 & 1.00 & 24.43 & 1.00 & 1 & 30.59 & 25.20 & 3247 & 10230 \\
\hline c7 & 1.03 & 28.26 & 37.26 & 23.82 & 1.01 & 49.50 & 1 & 25.13 & 106.1 & 1454 \\
\hline c8 & 1.00 & 3.43 & 1.44 & 1.00 & 1.00 & 1.97 & 1.21 & 1 & 128.9 & 406.0 \\
\hline c9 & 1.03 & 1.00 & 37.35 & 23.87 & 1.01 & 49.61 & 1.00 & 25.19 & 1 & 13.74 \\
\hline c10 & 1.05 & 1.05 & 8.36 & 5.49 & 1.05 & 11.40 & 1.05 & 5.79 & 1.05 & 1 \\
\hline
\end{tabular}

this paper. The approach was efficiently applied to both SISO and MIMO local uncertain system examples as well as to the performance analysis of the uncertain largescale system within the framework of hierarchical analysis approach. As a perspective for future work, one can expect an improvement, from conservatism point of view, for hierarchical analysis by combining different kinds of embedding.

\section{REFERENCES}

Braatz, R.D., Young, P.M., Doyle, J.C., and Morari, M. (1994). Computational complexity of $\mu$ calculation. IEEE Trans. Aut. Control, AC-39(5), 1000-1002.

Doyle, J. (1982). Analysis of feedback systems with structured uncertainties. IEE Proc., 129-D(6), 242-250.

Fan, M.K.H., Tits, A.L., and Doyle, J.C. (1991). Robustness in the presence of mixed parametric uncertainty and unmodeled dynamics. IEEE Trans. Aut. Control, $36(1), 25-38$.

Houpis, C. and Rasmussen, S. (1999). Quantitative feedback theory: fundamentals and applications. Control engineering. Marcel Dekker.

Iwasaki, T. and Hara, S. (1998). Well-posedness of feedback systems: Insights into exact robustness analysis and approximate computations. IEEE Trans. Aut. Control, 43, 619-630.

Korniienko, A., Scorletti, G., Colinet, E., and Blanco, E. (2011a). Control law design for distributed multi-agent systems. Technical report, Laboratoire Ampère, Ecole Centrale de Lyon. URL http: //hal . archives-ouvertes.fr/hal-00630543/en/.

Korniienko, A., Scorletti, G., Colinet, E., Blanco, E., Juillard, J., and Galayko, D. (2011b). Control law synthesis for distributed multi-agent systems: Application to active clock distribution networks. In IEEE American Control Conference, 4691- 4696. San Francisco.

Megretski, A. and Rantzer, A. (1997). System analysis via integral quadratic constraints. IEEE Trans. Aut. Control, 42(6), 819-830.

Safonov, M.G. (1980). Stability and Robustness of Multivariable Feedback Systems. MIT Press, Cambridge.

Safonov, M.G. (1983). Propagation of conic model uncertainty in hierarchical systems. IEEE Trans. Circuits and Systems, 388-396.

Scorletti, G. (1998). A more praticle formulation for robustness analysis. In IFAC Conference on System Structure and Control. Nantes, France.

Scorletti, G., Bombois, X., Barenthin, M., and Fromion, V. (2007). Improved efficient analysis for systems with uncertain parameters. In IEEE Conf. Decision and Control, 5038-5043. New Orleans.
Skogestad, S. and Postlethwaite, I. (2005). Multivariable Feedback Control, Analysis and Design. John Wiley and Sons Chischester.

Yaw, Y. and Balas, G. (2011). Development and application of an integrated framework for small uav flight control development. Mechatronics, 21(5), 789-802.

\section{APPENDIX: PROOF OF THEOREM 2}

The inclusion $G_{s} \subseteq G_{u}$ is equivalent to

$$
\forall \Delta_{s} \in \boldsymbol{\Delta}_{\mathbf{s}}, \bar{\sigma}\left(W_{o}^{-1}\left(\Delta_{s} \star M_{s}-G_{n o m}\right) W_{i}^{-1}\right)<1
$$

that is $\forall \Delta_{s} \in \boldsymbol{\Delta}_{\mathbf{s}}$,

$$
(.)^{*}\left[\begin{array}{cc}
W_{o}^{-*} W_{o}^{-1} & 0 \\
0 & -I
\end{array}\right]\left[\begin{array}{c}
\Delta_{s} \star M_{s}-G_{n o m} \\
W_{i}
\end{array}\right]<0
$$

Then the previous inequality holds if and only if there exists $\Phi \in \mathbb{C}^{\left(n_{o}+n_{i}\right) \times\left(n_{o}+n_{i}\right)}$ such that

$$
\forall \Delta_{s} \in \Delta_{\mathbf{s}},\left[\begin{array}{c}
\Delta_{s} \\
I
\end{array}\right]^{*} \Phi\left[\begin{array}{c}
\Delta_{s} \\
I
\end{array}\right] \geq 0
$$

and

$$
\begin{gathered}
{[.]^{*} \Phi\left[\begin{array}{cc}
I & 0 \\
A_{s} & B_{s}
\end{array}\right]} \\
+[.]^{*}\left[\begin{array}{cc}
W_{o}^{-*} W_{o}^{-1} & 0 \\
0 & -I
\end{array}\right]\left[\begin{array}{cc}
C_{s} & D_{s}-G_{n o m} \\
0 & W_{i}
\end{array}\right]<0
\end{gathered}
$$

With changes of variables $P_{i}=W_{i}^{*} W_{i}$ and $P_{o}=W_{o} W_{o}^{*}$, the last inequality can be rewritten

$$
\begin{gathered}
{[.]^{*} \Phi\left[\begin{array}{cc}
I & 0 \\
A_{s} & B_{s}
\end{array}\right]} \\
+\left[\begin{array}{cc}
0 & 0 \\
0 & -P_{i}
\end{array}\right]-(.)^{*}\left(-P_{o}^{-1}\right)\left[\begin{array}{ll}
C_{s} & D_{s}-G_{n o m}
\end{array}\right]<0
\end{gathered}
$$

Then, by Schur's lemma, this is equivalent to

$$
\left[\begin{array}{c|c}
-P_{o} & C_{s} D_{s}-G_{n o m} \\
\hline(.)^{*} & (.)^{*} \Phi\left[\begin{array}{cc}
I & 0 \\
A_{s} & B_{s}
\end{array}\right]+\left[\begin{array}{cc}
0 & 0 \\
0 & -P_{i}
\end{array}\right]
\end{array}\right]<0
$$

which after a factorization is equivalent to (5).

The invertibility of $W_{o}$ and $W_{i}$ are equivalent to the fact that $P_{o}$ and $P_{i}$ are positive definite. This concludes the proof of the First Solution.

The proof of the SECOND Solution i.e. (6) is obtained thanks to post and pre-multiplying inequality (.1) by bdiag $\left(\tilde{P}_{o}, I\right)=\operatorname{bdiag}\left(P_{o}^{-1}, I\right)=\operatorname{bdiag}\left(W_{o}^{-*} W_{o}^{-1}, I\right)$, change of variable $\tilde{P}_{n o m}=\tilde{P}_{o} G_{n o m}$ and further factorization. Finally, note that the invertibility of $W_{o}$ and $W_{i}$ are equivalent to the fact that $\tilde{P}_{o}$ and $P_{i}$ are positive definite. 